\title{
Growth of finite errors in ensemble prediction
}

\author{
M. Harle, F. Kwasniok, and U. Feudel \\ Institute for Chemistry and Biology of the Marine Environment, University of Oldenburg, Oldenburg, Germany \\ Received: 28 July 2005 - Revised: 18 January 2006 - Accepted: 18 January 2006 - Published: 12 June 2006
}

\begin{abstract}
We study the predictability of chaotic conservative and dissipative maps in the context of ensemble prediction. Finite-size perturbations around a reference trajectory are evolved under the full nonlinear system dynamics; this evolution is characterized by error growth factors and investigated as a function of prediction time and initial perturbation size. The distribution of perturbation growth is studied. We then focus on the worst-case predictability, i.e., the maximum error growth over all initial conditions. The estimate of the worst-case predictability obtained from the ensemble approach is compared to the estimate given by the largest singular value of the linearized system dynamics. For small prediction times, the worst-case error growth obtained from the nonlinear ensemble approach is exponential with prediction time; for large prediction times, a power-law dependence is observed the scaling exponent of which depends systematically on the initial error size. The question is addressed of how large an ensemble is necessary to reliably estimate the maximum error growth factor. A power-law dependence of the error in the estimate of the growth factor on the ensemble size is established empirically. Our results are valid for several markedly different chaotic conservative and dissipative systems, perhaps pointing to quite general features.
\end{abstract}

\section{Introduction}

Prediction is a major goal in many areas of science. The value of any prediction is greatly enhanced if information about the uncertainty associated with it is available. This issue is related to the dynamical predictability of a complex system. As is well known predictability of a dynamical system may vary enormously across state space. For example, different current weather conditions lead to predictions of different reliability.

Correspondence to: M. Harle

(m.harle@icbm.de)
Several methods for quantifying predictability are in use. Some of these are based on the linearized system dynamics. Characteristic numbers such as singular values of the linearized propagator or local Lyapunov exponents describe the evolution of infinitesimally small initial perturbations around a reference trajectory (Smith et al., 1999 and many references therein; Kalnay, 2002). The orientation of the initial error in state space is crucial. However, such methods cannot necessarily be expected to correctly capture the evolution of finite initial perturbations, particularly for large prediction times. The issue of finite initial errors can be tackled using ensembles, i.e., considering the evolution of an ensemble of initial perturbations of finite size under the full nonlinear dynamics of the system. Ensemble integrations are today routinely used in operational numerical weather forecasting (see Kalnay, 2002, for an overview). Again, the orientation of the initial perturbations is of crucial importance. The directions of fastest error growth are of particular importance since they limit predictability. Two different methods of initialising the ensemble have been established. Some groups use the fastest growing directions of the linearized system, the singular vectors, as initial errors for the ensemble integrations (Buizza, 1996); others prefer a more involved procedure called breeding based on the full nonlinear system to initialise the errors (Toth and Kalnay, 1993, 1997). Recently, the structure of local bred vectors in geographical space has been investigated and used to define a local dimension of atmospheric flow which, in some sense, carries information on local predictability (Patil et al., 2001). A connection between spatial degrees of freedom and prediction and predictability has also been found earlier (Fraedrich et al., 1995). Furthermore, conditional nonlinear optimal perturbations have been studied and compared with singular vectors in order to assess the importance of the nonlinearity and to find initial perturbations that will most probably lead to a certain future state (Mu et al., 2003).

In the present paper, we study some basic problems associated with the evolution of finite-size errors in nonlinear

Published by Copernicus GmbH on behalf of the European Geosciences Union and the American Geophysical Union. 
dynamical systems. We restrict ourselves to low-dimensional chaotic maps here which allow the investigation of very large ensembles and large prediction times. We discuss the error growth in ensemble prediction as a function of prediction time and initial perturbation size. Moreover, we address the question of how large an ensemble is needed to obtain reliable estimates of the error growth.

The paper is organised as follows: In Sect. 2 we introduce the concept of ensembles, as well as the quantities that are later used to quantify the predictability of the initial state. Section 3 gives a brief overview of the model systems studied, followed by the results in Sect. 4. In particular, we will show that the error growth in the worst case depends on prediction time in a systematic way. Furthermore, the distributions of error growth factors converge with a characteristic dependence on ensemble size, a fact that can help to determine the minimal ensemble size needed for a given error margin. We close with a discussion in Sect. 5.

\section{Ensemble prediction and error growth factors}

In ensemble studies, not only a single trajectory of the system is followed over a certain period of time, but a whole bunch, or ensemble, of trajectories. These are typically initialised at some time $t_{0}$ around the current state vector $\boldsymbol{x}\left(t_{0}\right)$ of the reference trajectory (also called the "true" trajectory):

$\tilde{\boldsymbol{x}}_{j}\left(t_{0}\right)=\boldsymbol{x}\left(t_{0}\right)+\boldsymbol{\varepsilon}_{j}, \quad j=1, \ldots, N_{\mathrm{ens}}$

$N_{\text {ens }}$ is the size of the ensemble, $\tilde{\boldsymbol{x}}_{j}\left(t_{0}\right)$ denote the initial state vectors of the ensemble members and $\boldsymbol{\varepsilon}_{j}$ are the initial separations from the reference trajectory. All states, the reference as well as all ensemble members, evolve according to the full nonlinear system dynamics. After some prediction time $\tau$, for each ensemble member the error growth factor $m_{j}^{(\tau)}$ is calculated as the ratio between the distance from the reference trajectory and the initial distance:

$m_{j}^{(\tau)}\left(\boldsymbol{x}\left(t_{0}\right)\right)=\frac{\left\|\tilde{\boldsymbol{x}}_{j}\left(t_{0}+\tau\right)-\boldsymbol{x}\left(t_{0}+\tau\right)\right\|}{\left\|\tilde{\boldsymbol{x}}_{j}\left(t_{0}\right)-\boldsymbol{x}\left(t_{0}\right)\right\|}$

Distances are measured in the Euclidian metric throughout this paper. The error growth factors are used to characterize the predictability of the state $\boldsymbol{x}\left(t_{0}\right)$. To this end, the minimum, maximum and average growth factor $m_{\min }^{(\tau)}\left(\boldsymbol{x}\left(t_{0}\right)\right)$, $m_{\max }^{(\tau)}\left(\boldsymbol{x}\left(t_{0}\right)\right)$ and $m_{\text {avg }}^{(\tau)}\left(\boldsymbol{x}\left(t_{0}\right)\right)$ of the ensemble are considered. To simplify the notation, we will from now on suppress the explicit dependence of the error growth factors $m_{j}^{(\tau)}$ on $\boldsymbol{x}\left(t_{0}\right)$. The maximal error growth factor $m_{\max }^{(\tau)}$ obviously describes the worst case regarding predictability over the time $\tau$. Correspondingly, $m_{\min }^{(\tau)}$ stands for the best case, and $m_{\text {avg }}^{(\tau)}$ can be interpreted as the error growth one should expect on average.

There are a number of free parameters to be set, some of which will be varied in order to study the dependence of predictability on their values. All results presented in Sect. 4 were obtained with a particular initial distribution of ensemble states. Namely, all initial separations are of the same size, $d_{0}$, the direction in state space is random with equal probability for all directions. Other initial distributions were compared against this choice: firstly, a uniformly random separation size $\leq d_{0}$, and secondly Gaussian distributions with standard deviation $d_{0}$ in all coordinates, both with uniformly random direction. These alternative ways of initialising the ensemble were found to lead to almost the same distributions of the error growth factors $m$ and are therefore not discussed in this study. In the case of high-dimensional models of real-world processes such as numerical weather prediction, certain directions of initial error can be more probable than others and the initial distribution of the ensemble should be changed accordingly.

The initial perturbation size $d_{0}$, together with the time horizon $\tau$, are parameters that are varied to study predictability. It turned out that both of them have a strong influence on the predictability. In practice, the size $N_{\text {ens }}$ of the ensemble is very important since it is limited by the available computing power. Therefore, results for different values of $N_{\text {ens }}$ are compared with each other to determine a minimal value of $N_{\text {ens }}$ needed to ensure a reasonable estimate of $m_{\max }^{(\tau)}$, for example.

\section{Model systems}

The dynamical systems studied have to be simple enough to remain feasible even for large ensembles and long prediction times. On the other hand, they ought to show typical chaotic behaviour. Based on these premises, we chose twodimensional chaotic maps. The standard map is a conservative system well studied in the context of Hamiltonian chaos (Lichtenberg and Lieberman, 1992; Chirikov, 1979):

$$
\begin{array}{ll}
x_{1}\left(t_{n+1}\right)=x_{1}\left(t_{n}\right)+x_{2}\left(t_{n}\right) & (\bmod 2 \pi) \\
x_{2}\left(t_{n+1}\right)=x_{2}\left(t_{n}\right)+K \sin \left(x_{1}\left(t_{n+1}\right)\right) & (\bmod 2 \pi) .
\end{array}
$$

The phase space of this sytem consists of islands of regular motion and layers of chaotic motion between the closed curves of regular motion. The relative size of the chaotic layer depends on the nonlinearity parameter $K$. We investigate this system for two extreme cases: at $K=0.6$, a value for which the chaotic layer is occupying a very small portion of state space, and at $K=4.2$, with the chaotic layer spread out over a large part of state space. The trajetory is always initialised in the chaotic layer. The dynamics of this system are shown in Fig. 1 for the parameter values considered here.

The quasiperiodicity map (Grebogi et al., 1985) is more involved and has the form

$$
\begin{aligned}
& x_{1}\left(t_{n+1}\right)=x_{1}\left(t_{n}\right)+c_{1}+c_{3} p_{1}\left(\boldsymbol{x}\left(t_{n}\right)\right) \\
& x_{2}\left(t_{n+1}\right)=x_{2}\left(t_{n}\right)+c_{2}+c_{3} p_{2}\left(\boldsymbol{x}\left(t_{n}\right)\right)
\end{aligned}
$$

where we use the parameter sets $c_{1}=0.2, c_{2}=0.3, c_{3}=0.6$ and $c_{1}=0.42, c_{2}=0.3, c_{3}=1.6$. For these parameter sets, and for 

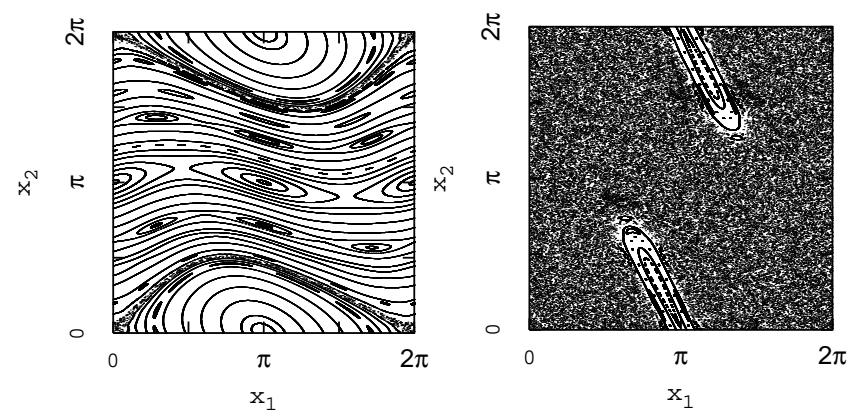

Fig. 1. Dynamics of the standard map at $K=0.6$ (left panel) and $K=4.2$ (right panel).
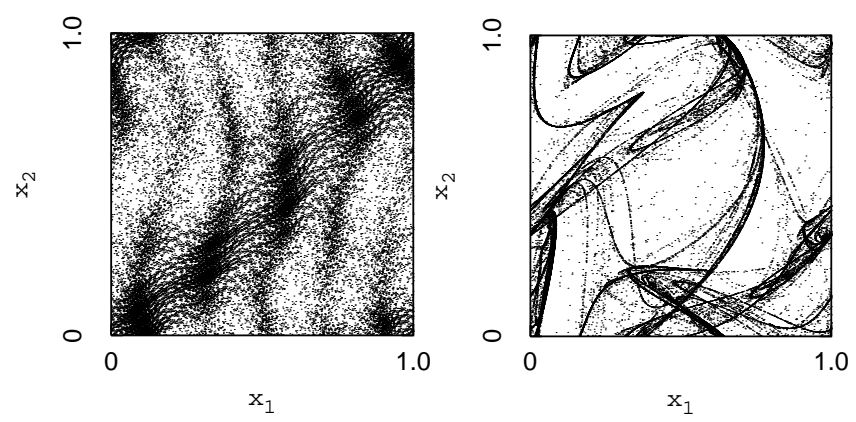

Fig. 2. Dynamics of the quasiperiodicity map at parameter values of $c_{1}=0.2, c_{2}=0.3, c_{3}=0.6$ (left panel) and $c_{1}=0.42, c_{2}=0.3$, $c_{3}=1.6$ (right panel).

the expressions for $p_{1}$ and $p_{2}$ given below, the quasiperiodicity map is dissipative. For the first parameter values the system is only very weakly chaotic, while in the second case it is considerably more chaotic and also more dissipative. Note that parameter $c_{3}$ determines the strength of the nonlinearity. An overview of the dynamics can be seen in Fig. 2 .

The nonlinearities $p_{1}$ and $p_{2}$ are given by

$$
\begin{aligned}
& p_{1}\left(x\left(t_{n}\right)\right)=\frac{1}{2 \pi}\left(A_{1} \sin \left(2 \pi\left(x_{1}\left(t_{n}\right)+K_{1}\right)\right)+\right. \\
& A_{2} \sin \left(2 \pi\left(x_{2}\left(t_{n}\right)+K_{2}\right)\right)+ \\
& A_{3} \sin \left(2 \pi\left(x_{1}\left(t_{n}\right)+x_{2}\left(t_{n}\right)+K_{3}\right)\right)+ \\
& \left.A_{4} \sin \left(2 \pi\left(x_{1}\left(t_{n}\right)-x_{2}\left(t_{n}\right)+K_{4}\right)\right)\right), \\
& p_{2}\left(\boldsymbol{x}\left(t_{n}\right)\right)=\frac{1}{2 \pi}\left(B_{1} \sin \left(2 \pi\left(x_{1}\left(t_{n}\right)+J_{1}\right)\right)+\right. \\
& B_{2} \sin \left(2 \pi\left(x_{2}\left(t_{n}\right)+J_{2}\right)\right)+ \\
& B_{3} \sin \left(2 \pi\left(x_{1}\left(t_{n}\right)+x_{2}\left(t_{n}\right)+J_{3}\right)\right)+ \\
& \left.B_{4} \sin \left(2 \pi\left(x_{1}\left(t_{n}\right)-x_{2}\left(t_{n}\right)+J_{4}\right)\right)\right) \text {. }
\end{aligned}
$$

The values of the constants $A_{i}, K_{i}, B_{i}, J_{i}$ are shown in Table 1.

At these parameter values, there exists a chaotic layer covering almost the whole state space of the system. This, along with its structure that is substantially more complex than that
Table. Parameter values used for the quasiperiodicity map.

\begin{tabular}{crc}
\hline$i$ & \multicolumn{1}{c}{$A_{i}$} & $K_{i}$ \\
\hline 1 & -0.2681366365 & 0.9854608430 \\
2 & -0.9106755940 & 0.5044604561 \\
3 & 0.3117202638 & 0.9470747252 \\
4 & -0.0400397884 & 0.2335010550 \\
\hline$i$ & \multicolumn{1}{c}{$B_{i}$} & $J_{i}$ \\
\hline 1 & 0.0881861167 & 0.9903072286 \\
2 & -0.5650288998 & 0.3363069701 \\
3 & 0.1629954873 & 0.2980492123 \\
4 & -0.8039888198 & 0.1550646728 \\
\hline
\end{tabular}

of the standard map, made us choose this system for our investigation. Another reason for comparing these systems will become clear in the next section. Furthermore, comparing different systems allows one to draw more general conclusions than considering only one possibly special case.

The modulo operations are only applied once the distances of ensemble states from the true state have been calculated. This ensures that no artefacts are produced by shifting some ensemble members that happened to cross the boundary of the unit cell.

\section{Results}

In this section, we compare distributions of error growth factors $m^{(\tau)}$ as defined in Sect. 2 and extract information about the dependence of the worst-case predictability on prediction time $\tau$ and initial error $d_{0}$. Also, we show a general convergence behaviour of these distributions and present results for the minimal ensemble size $N_{\mathrm{ens}}^{\min }$ needed for a given convergence level, i.e., a given error margin $\epsilon$.

The initial states $\boldsymbol{x}_{0}$ contributing to the distributions of error growth factors are obtained by chopping up a long trajectory ( $10^{5}$ iterations) into consecutive pieces of length $\tau$. Thus, the number of initial states for a prediction time horizon of $\tau$ is $10^{5} / \tau$. All results are based on the same initial distributions for each $\tau$.

The maximum error growth factor over the ensemble, $m_{\max }^{(\tau)}$, is arguably the most important of the statistical quantities considered, since it is the one that limits predictability (Ziehmann et al., 2000). Large enough an ensemble is required to obtain a good estimate of $m_{\max }^{(\tau)}$. It will be shown below that the quantities $m_{\max }^{(\tau)}, m_{\min }^{(\tau)}$ and $m_{\text {avg }}^{(\tau)}$ - that can all be regarded as measures of predictability - are not equally costly to determine up to a specified error margin.

\subsection{Distributions of error growth factors}

After calculating the error growth factors for many initial states in the chaotic layer, one can get a first impression of 

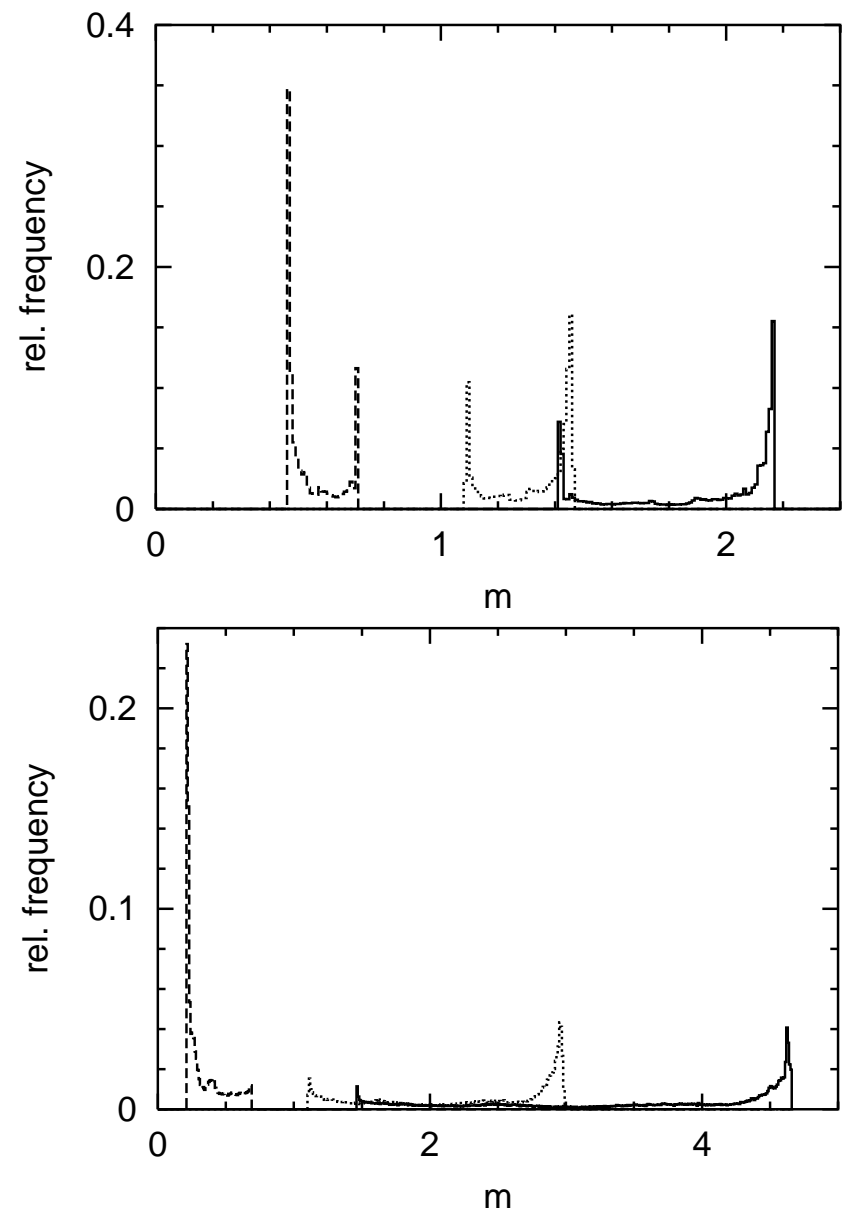

Fig. 3. Standard map $(K=0.6)$ : Distributions of error growth factors $m_{\max }^{(\tau)}$ (solid), $m_{\min }^{(\tau)}$ (dashed) and $m_{\text {avg }}^{(\tau)}$ (dotted) for $\tau=1$ (top) and $\tau=2$ (bottom). Ensemble size is $N_{\mathrm{ens}}=20000$; initial perturbation size is $d_{0}=0.001$.

the occurring values by looking at the distribution of $m_{\min }^{(\tau)}$, $m_{\text {avg }}^{(\tau)}$ and $m_{\max }^{(\tau)}$. The corresponding histograms are shown in Fig. 3 for the standard map and in Fig. 4 for the quasiperiodicity map. In the case of the standard map the distribution of $m_{\min }^{(\tau)}$ does not change its general structure when going from $\tau=1$ to $\tau=2$, but the small values become more frequent. Likewise, the high values of $m_{\max }^{(\tau)}$ increase in weight. The distributions of $m_{\mathrm{avg}}^{(\tau)}$ and $m_{\max }^{(\tau)}$ generally become broader and flatter and are shifted towards higher values when increasing the prediction time. For the quasiperiodicity map the broadening and shifting is much more pronounced for $m_{\text {avg }}^{(\tau)}$ and $m_{\max }^{(\tau)}$. For $m_{\min }^{(\tau)}$, the same behaviour can be seen as in the standard map, small values are found more often.

These figures clearly show an important difference between the two systems: The distributions for the standard map have very sharp peaks near the ends of the distributions.
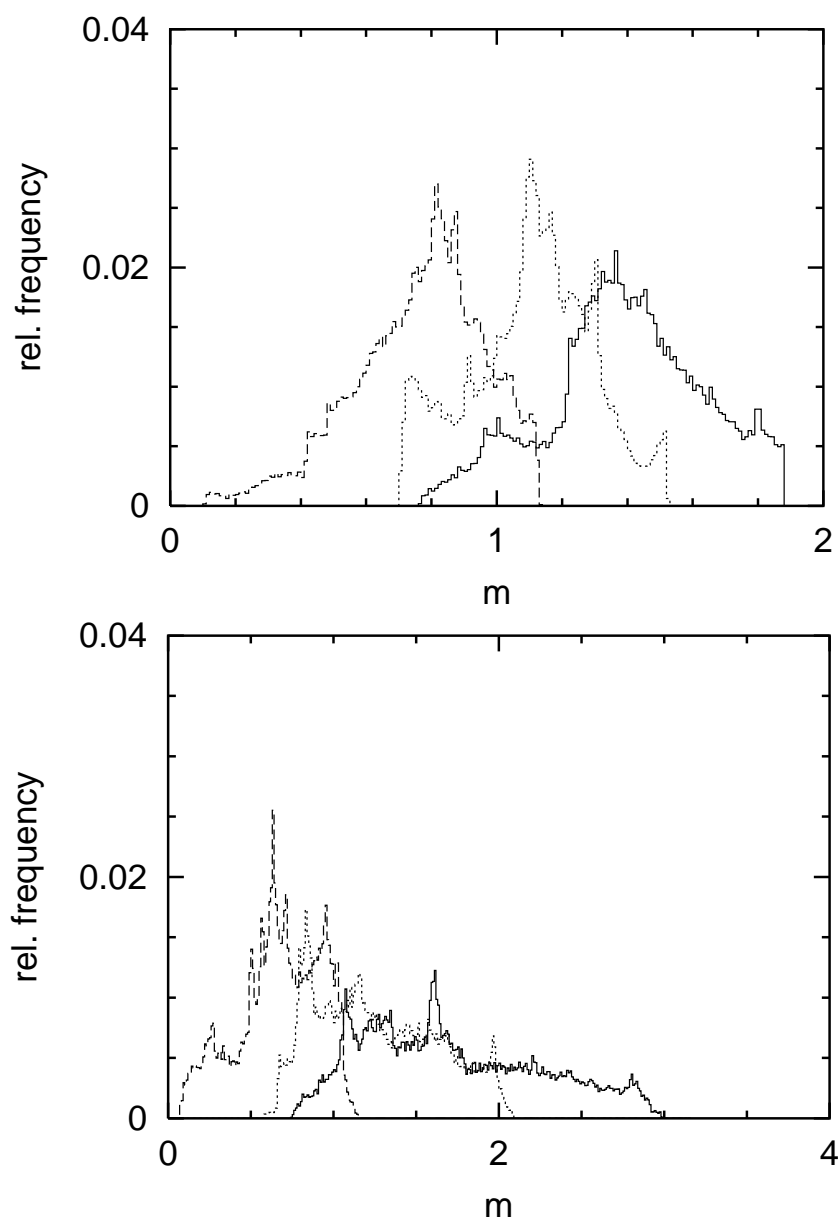

Fig. 4. As Fig. 3, but for the quasiperiodicity map $\left(c_{1}=0.2\right.$, $\left.c_{2}=0.3, c_{3}=0.6\right)$.

On the other hand, the quasiperiodicity map exhibits more Gaussian-like distributions, cut off by $m=0$ in the case of $m_{\min }^{(\tau)}$. This difference expresses a fundamental difference of the underlying dynamical systems, enabling us to draw general conclusions not restricted to the particular systems under investigation.

It should be noted that for the standard map, the size of the system is $\ell_{\mathrm{Std}}=2 \pi$, whereas for the quasiperiodicity map $\ell_{\mathrm{Qp}}=1$. Therefore, initial errors of the same absolute size $d_{0}$ have different relative sizes for the different systems.

4.2 Dependence of the worst-case error growth on prediction time and initial perturbation size

We now focus on the right end of the distribution of $m_{\max }^{(\tau)}$, i.e., the largest error growth factor found when comparing all initial positions, $M^{(\tau)}$ :

$M^{(\tau)}=\max _{\boldsymbol{x}\left(t_{0}\right)} m_{\max }^{(\tau)}\left(\boldsymbol{x}\left(t_{0}\right)\right)$. 


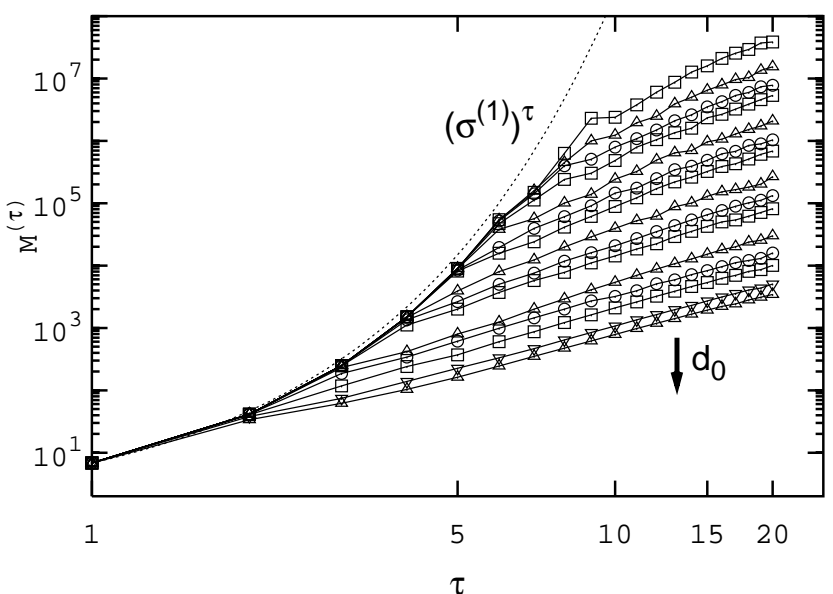

Fig. 5. Standard map $(K=4.2)$ : Dependence of $M^{(\tau)}$ on $\tau$. The curves represent different values of $d_{0}$, from $10^{-4}$ (top) to $3 \times 10^{-1}$ (bottom). The dotted line shows an exponential increase, see text for details. $\tau$ is shown on a logarithmic scale.

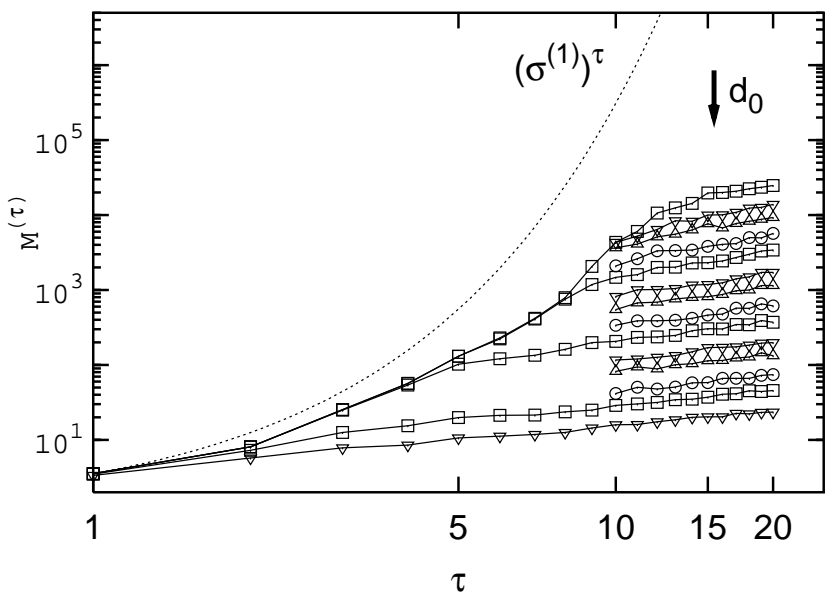

Fig. 6. As Fig. 5, but for the quasiperiodicity map $\left(c_{1}=0.42\right.$, $c_{2}=0.3, c_{3}=1.6$ ). Here, the values of $d_{0}$ range from $10^{-4}$ (top) to $2 \times 10^{-1}$ (bottom). For some values of $d_{0}, M^{(\tau)}$ was only calculated for $10 \leq \tau \leq 20$.

This quantity represents the worst case for a prediction of time $\tau$ when the initial state is not known. The dependence of $M^{(\tau)}$ on $\tau$ is shown in Fig. 5 for the standard map and in Fig. 6 for the quasiperiodicity map. The plots clearly show an exponential increase for small values of $\tau$.

For small initial errors $d_{0}$ and short times $\tau$ one expects a growth of $M^{(\tau)}$ according to the singular value $\sigma_{1}^{(\tau)}$ of the linear propagator over $\tau$ iterations $\mathbf{J}^{\tau}$ :

$\sigma_{i}^{(\tau)}\left(\boldsymbol{x}\left(t_{0}\right)\right)=\left\|\mathbf{J}^{\tau}\left(\boldsymbol{x}\left(t_{0}\right)\right) \boldsymbol{v}_{i}^{(\tau)}\left(\boldsymbol{x}\left(t_{0}\right)\right)\right\|$

where $\mathbf{J}^{\tau}$ is the product of Jacobians along the trajectory and $\boldsymbol{v}_{i}^{(\tau)}\left(\boldsymbol{x}\left(t_{0}\right)\right)$ are the right singular vectors with moduli 1. For
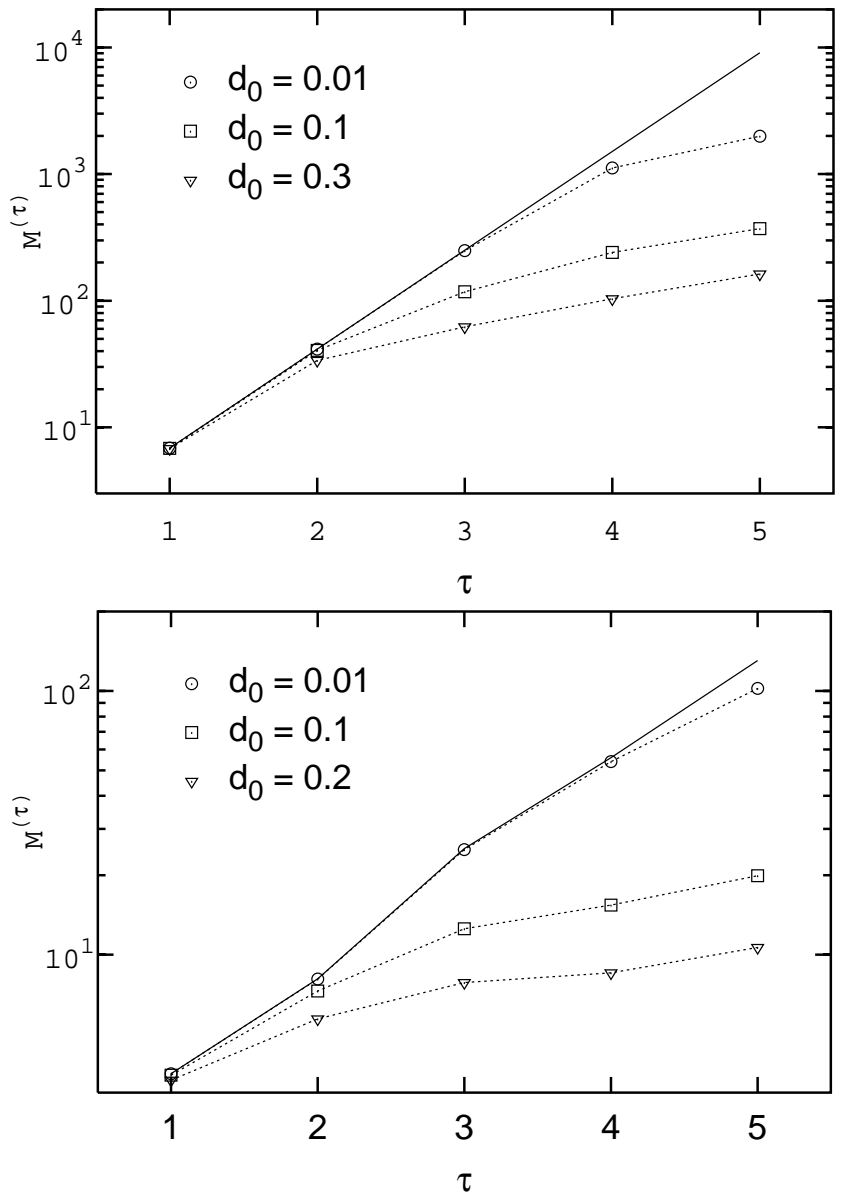

Fig. 7. Comparison of $M^{(\tau)}$ (symbols) and $\sigma^{(\tau)}$ (solid line) for the standard map ( $K=4.2$; upper panel) and the quasiperiodicity map $\left(c_{1}=0.42, c_{2}=0.3, c_{3}=1.6\right.$; lower panel $)$.

comparison, the dotted line in Figs. 5 and 6 shows $\left(\sigma^{(1)}\right)^{\tau}$, where $\sigma^{(1)}$ is the largest singular value of the linearized map for one time step. This means that for $\tau=1$, the error grows at most by a factor of $\sigma^{(1)}$, provided the linearisation is valid. If, after one iteration, the error lies in the direction of maximal error growth of the new state $\boldsymbol{x}\left(t_{0}+1\right)$, the growth over two iterations will be $\left(\sigma^{(1)}\right)^{2}$. Thus, the fact that the solid lines are below the dotted lines in Figs. 5 and 6 seems reasonable, because errors will very rarely happen to point in the least predictable directions over several iterations.

Actually, it is more appropriate to compare $M^{(\tau)}$ with $\sigma^{(\tau)}$, the largest singular value when linearizing the map over $\tau$ steps. This gives an indication of where the linearisation breaks down. For example, in the case of the quasiperiodicity map this happens at $\tau=4$ or $\tau=5$ for $d_{0}=10^{-2}$ but already at $\tau=2$ for $d_{0}=10^{-1}$ (Fig. 7). The influence of the nonlinearity does not lead to an increase of error growth. In fact the errors do not grow as fast as in the linear system, which can already 

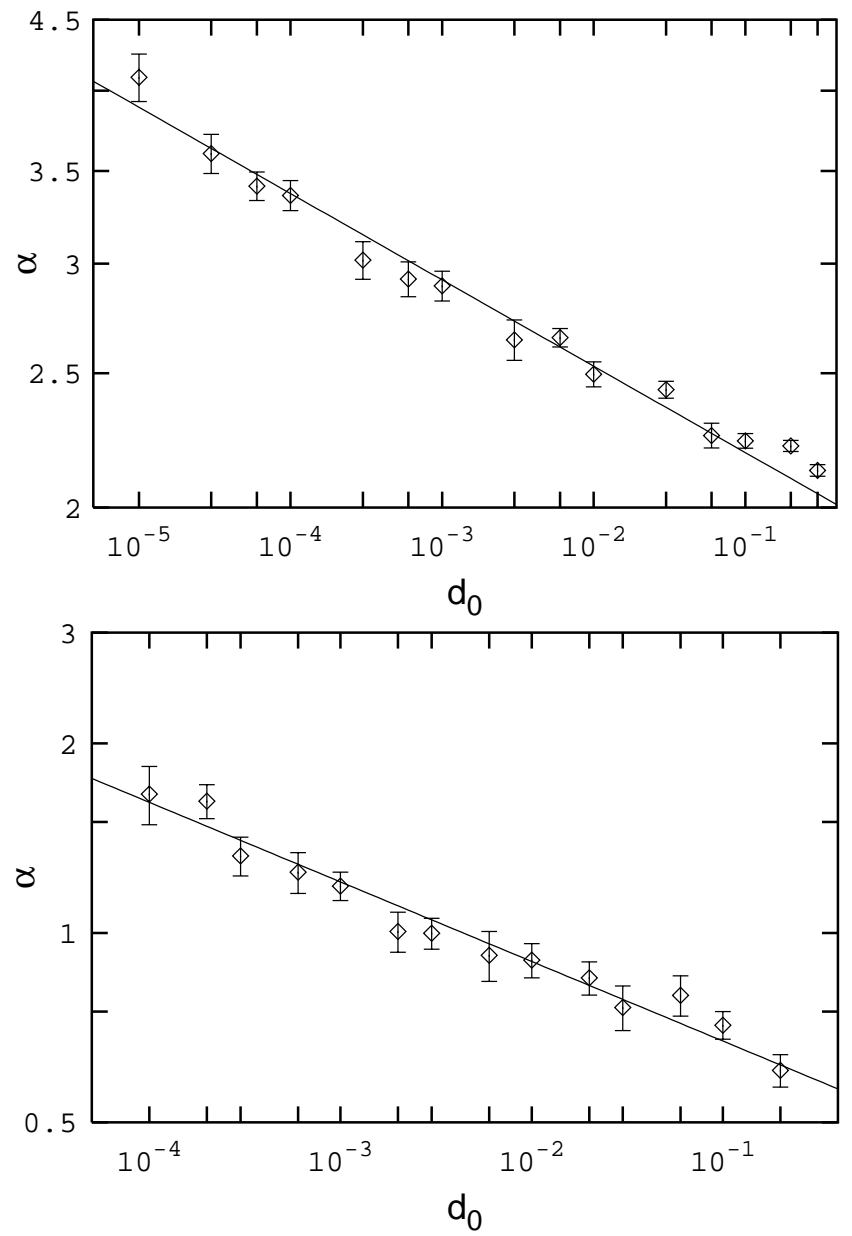

Fig. 8. Double-logarithmic plot of scaling exponent $\alpha$ of $M^{(\tau)}$ for large $\tau$. Results for the standard map ( $K=4.2$; upper panel) and the quasiperiodicity map $\left(c_{1}=0.42, c_{2}=0.3, c_{3}=1.6\right.$; lower panel), based on Figs. 5 and 6. Linear least-squares fits were made for $\tau \geq 10$ in those figures, except for the quasiperiodicity map at $d_{0}=10^{-4}$ where only $\tau \geq 12$ were used; error bars represent one standard deviation.

be guessed from looking at Figs. 5 and 6 . This is due to the folding in state space that occurs because of the nonlinearity.

The behaviour of $M^{(\tau)}$ for large times is qualitatively different. The double-logarithmic plots of Figs. 5 and 6 suggest a power-law

$M^{(\tau)} \sim \tau^{\alpha}$.

Values of the exponent $\alpha$ were obtained from the interval $\tau \in$ $[10,20]$ (except for the smallest $d_{0}$ in the quasiperiodicity map) and are shown in Fig. 8. A power-law dependence of $\alpha$ on $d_{0}$ is found to agree well with the data.

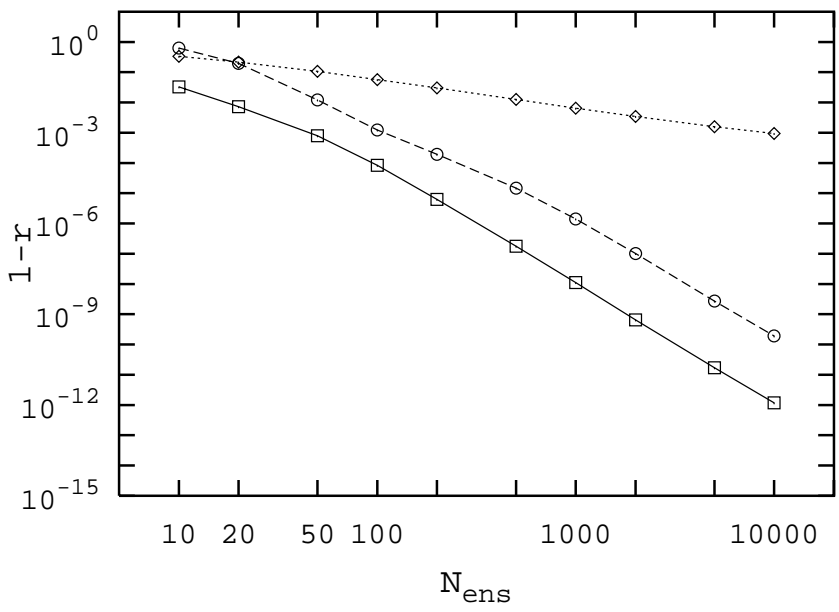

Fig. 9. Standard map $(K=0.6)$ : Convergence of estimates of $m_{\max }^{(\tau)}$ (squares), $m_{\text {avg }}^{(\tau)}$ (diamonds) and $m_{\min }^{(\tau)}$ (circles) with ensemble size characterized by linear correlation $r . \tau=1 ; d_{0}=0.2$.

\subsection{Minimal ensemble size}

In order to define a minimal size $N_{\mathrm{ens}}^{\min }$ of an ensemble, one needs a quantitative measure of how close the results for a given ensemble size $N_{\text {ens }}$ are to the hypothetical results for an infinite ensemble. This $N_{\mathrm{ens}}^{\min }$ will of course depend on the prediction time $\tau$ and the size of the initial error $d_{0}$. In the following, we will use the largest ensemble $\left(N_{\text {ens }}=20000\right)$ as a reference, because it is our best approximation to an infinite ensemble. Results for smaller ensembles are judged by how close they are to the reference. Measurement of this closeness or "convergence" of ensembles is done in two ways, one using the full spatially-resolved data, and one using histograms.

The first approach looks at each initial state $\boldsymbol{x}_{0}$ and compares results for, say, $m_{\max }^{(\tau), N_{\text {ens }}}\left(\boldsymbol{x}_{0}\right)$ obtained by using different ensemble sizes with the corresponding value for the reference ensemble, $\hat{m}_{\max }^{(\tau)}\left(\boldsymbol{x}_{0}\right)=m_{\max }^{(\tau), N_{\text {ens }}=20000}\left(\boldsymbol{x}_{0}\right)$.

Measurement of convergence of the ensemble is done by calculating the linear correlation $r$ between $m_{\max }^{(\tau), N_{\mathrm{ens}}}\left(\boldsymbol{x}_{0}\right)$ and $\hat{m}_{\max }^{(\tau)}\left(\boldsymbol{x}_{0}\right)$ for all $\boldsymbol{x}_{0}$, giving an ideal value of $r=1$ for exactly proportional data sets and $r=0$ for uncorrelated data sets. Thus, $1-r$ is the error introduced by using a finite ensemble size. Its dependence on $N_{\text {ens }}$ is shown in Figs. 9 and 10 for the standard map at $K=0.6$ and $K=4.2$, respectively, and for the quasiperiodicity map in Figs. 11 and 12. For ensembles that are large enough, a power-law decrease of $1-r$ can be seen in all cases. For $m_{\text {avg }}^{(\tau)}$, this is valid over the entire range of $N_{\mathrm{ens}}$, but the error $1-r$ is typically orders of magnitude larger than the errors for $m_{\min }^{(\tau)}$ and $m_{\max }^{(\tau)}$.

If the ensemble is very small, the error of $m_{\min }^{(\tau)}$ can remain almost constant at a high value, especially in the case of the 


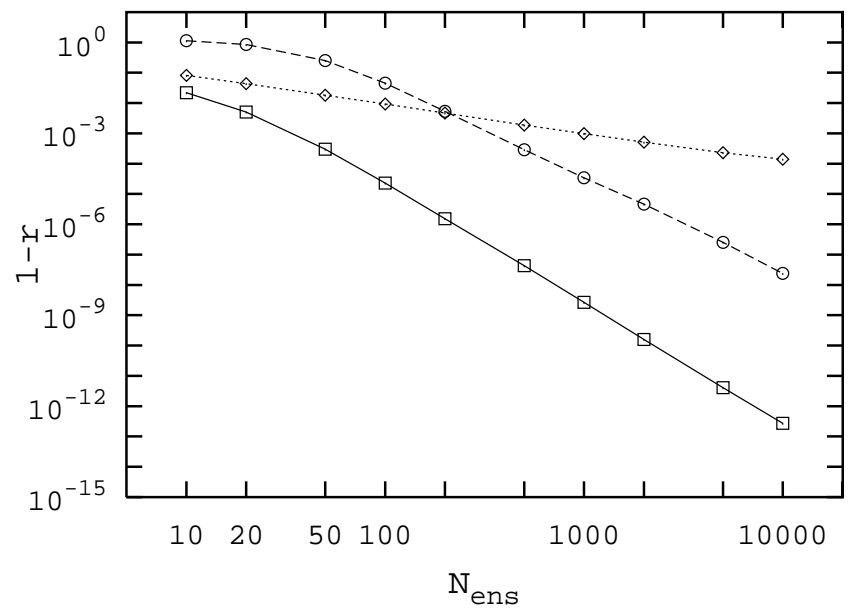

Fig. 10. As Fig. 9, but for the standard map at $K=4.2$.

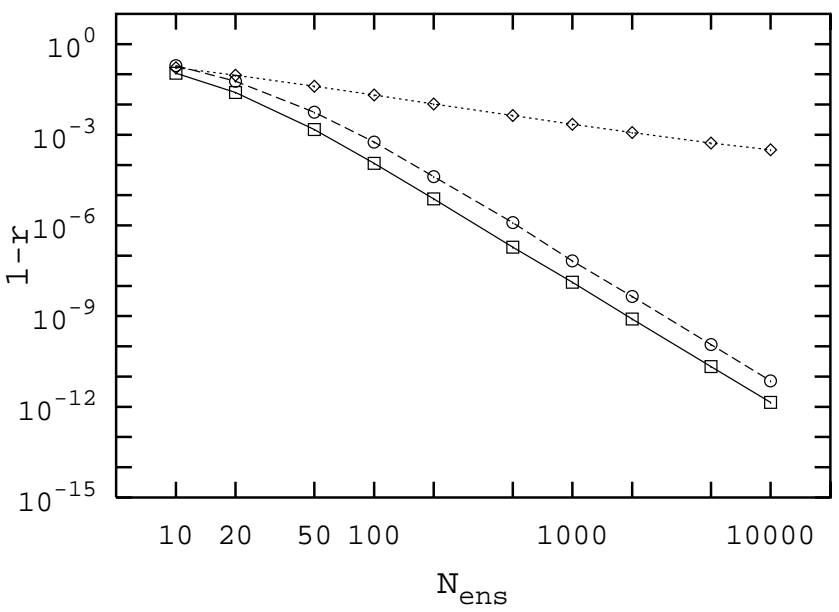

Fig. 11. As Fig. 9, but for the quasiperiodicity map $\left(c_{1}=0.2\right.$, $c_{2}=0.3, c_{3}=0.6$ ).

standard map at $K=4.2$. The range of ensemble sizes for which this behaviour occurs extends towards increasing $N_{\text {ens }}$ for larger times $\tau$ (not shown), whereas it depends only very weakly on $d_{0}$. In the quasiperiodicity map, this almost constant error of $m_{\min }^{(\tau)}$ can never be found as pronounced as in the case of the standard map.

We find that when the initial errors are small, only $m_{\min }^{(\tau)}$ depends considerably on $\tau$. This is most likely due to the dependence of the error growth factors $m$ on the initial perturbation direction in state space. If the smallest value $m_{\min }^{(\tau)}$ is attained only for a narrow interval of initial directions, small ensembles can be expected to produce a bad estimate of $m_{\min }^{(\tau)}$. When initial errors are not small, $m_{\max }^{(\tau)}$ also shows a strong dependence on $\tau$. This comes from the fact that longer prediction times together with larger initial errors create many more peaks in the distribution of error growth fac-

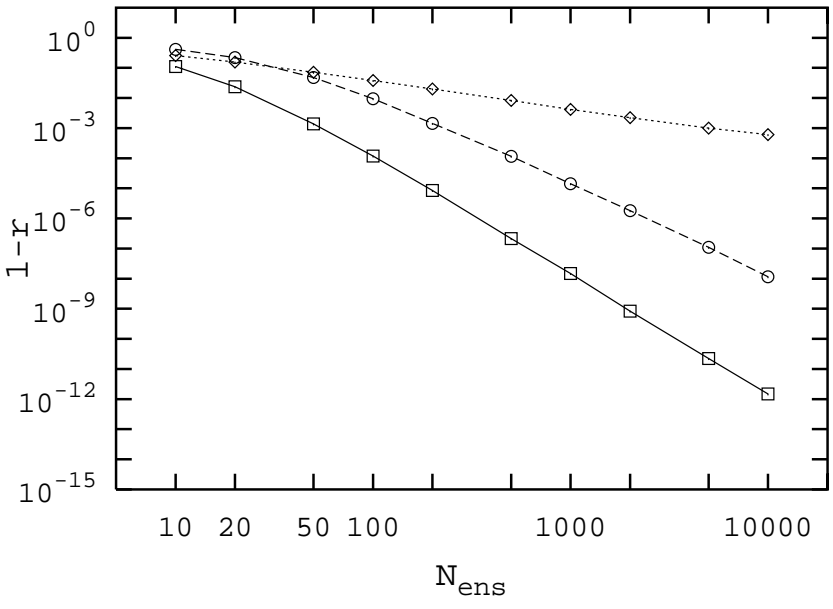

Fig. 12. As Fig. 9, but for the quasiperiodicity map $\left(c_{1}=0.42\right.$, $\left.c_{2}=0.3, c_{3}=1.6\right)$.

tors $m$ over initial error orientation. Therefore it becomes more difficult to find the global maximum, i.e., $m_{\max }^{(\tau)}$.

The power-law behaviour mentioned above, obeyed for large $N_{\text {ens }}$, can be expressed as a scaling exponent $\gamma$ :

$1-r \sim N_{\mathrm{ens}}^{\gamma}$

This exponent is extracted from the data shown in Figs. 912 , for example, by applying a linear least squares fit over a suitable interval of $N_{\text {ens }}$. The values of $\gamma$ for $m_{\text {avg }}^{(\tau)}$ and $m_{\max }^{(\tau)}$ are plotted in Fig. 13 for $\tau=1$ and $\tau=5$. We find that for $m_{\text {avg }}^{(\tau)}$, the scaling exponent $\gamma$ is constant and close to -1 for a wide range of initial errors $d_{0}$ and prediction times $\tau$. For $m_{\max }^{(\tau)}$, however, the behaviour for larger times is different, showing an increase for already quite large errors $d_{0}$ in the case of the standard map. In these cases, $1-r$ does not reach as low values as for smaller $d_{0}$, so the higher values of $\gamma$ can be the result of incomplete convergence towards the asymptotic behaviour. For the quasiperiodicity map, $m_{\max }^{(\tau)}$ remains close to -4 for almost the whole range of $d_{0}$, even up to higher values of $\tau$. One has to keep in mind that fitting a straight line to the data is not straightforward if $d_{0}$ and $\tau$ are too large. The error bars in Fig. 13 reflect this uncertainty.

The second approach to measuring the convergence of the results regarding the ensemble size does not use the spatial information contained in the data. This means that the values of $m_{\max }^{(\tau)}$, for example, are not associated with the state to which they originally belong. Instead, the data is stored in histograms. These can then be compared for different ensemble sizes $N_{\text {ens }}$, where the largest ensemble is again used as a reference. In order to determine the convergence of the ensemble, we calculate the relative entropy $s_{\text {rel }}$ between pairs of histograms. The relative entropy of a distribution $p(m)$ 

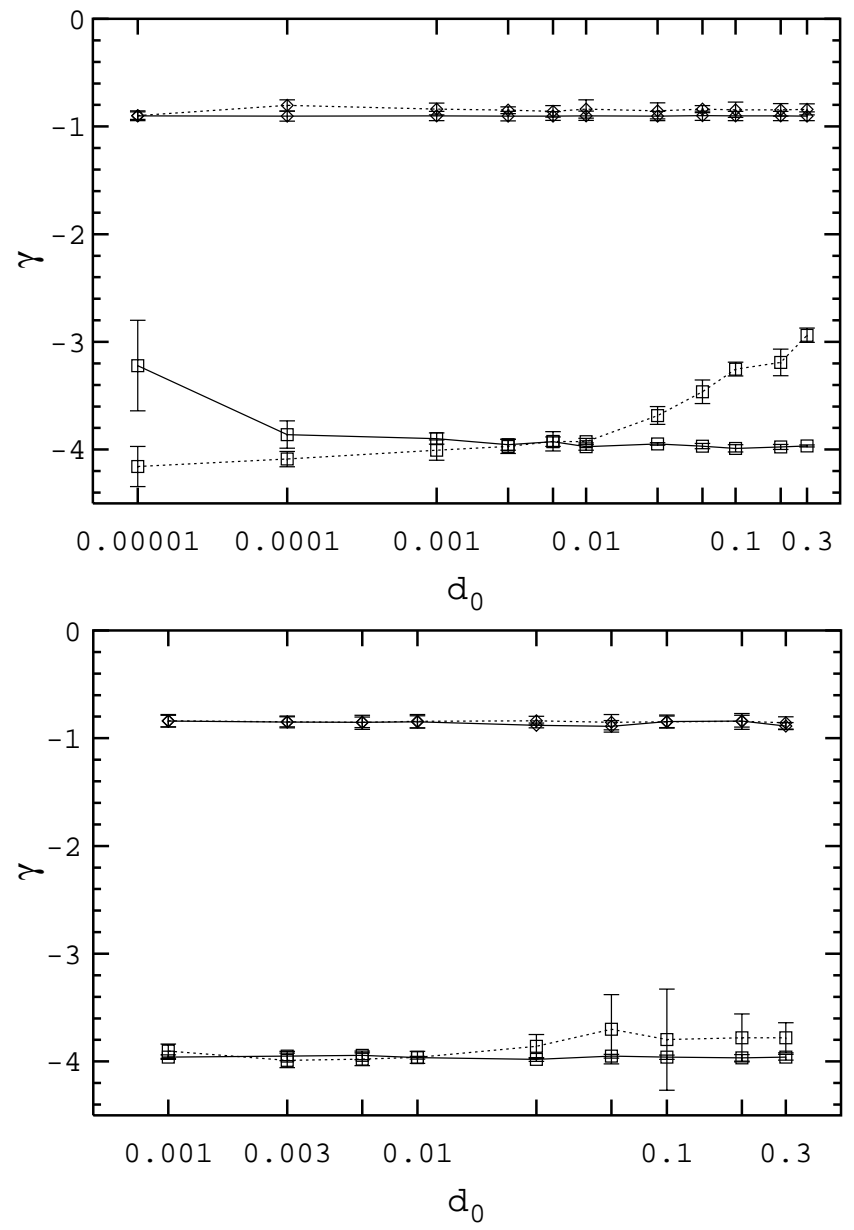

Fig. 13. Scaling exponent $\gamma$ of $1-r$ for large $N_{\text {ens }}$. Top panel is for the standard map ( $K=4.2$ ), bottom panel for the quasiperiodicity map $\left(c_{1}=0.42, c_{2}=0.3, c_{3}=1.6\right)$. The points show $m_{\max }^{(\tau)}$ (squares) and $m_{\mathrm{avg}}^{(\tau)}$ (diamonds), solid lines connect points for $\tau=1$, dotted lines for $\tau=5$. Error bars indicate two standard deviations.

with respect to a reference distribution $q(m)$ is defined as

$s_{\text {rel }}(p \mid q)=-\int_{0}^{\infty} \mathrm{d} m p(m) \ln \frac{p(m)}{q(m)}$,

where in our case $q(m)$ is the reference histogram of a large ensemble, and $p(m)$ is the histogram for a smaller ensemble size. The relative entropy can be interpreted as the amount of information lost due to the finiteness of the ensemble. It is widely used and has a sound theoretical basis (Honerkamp, 1994). For identical distributions a value of $s_{\text {rel }}=0$ is obtained. The results for the standard map are shown in Fig. 14 (cf. Fig. 9 for the corresponding linear correlation data). The analogous graphs for the quasiperiodicity map are plotted in Fig. 15 (cf. Fig. 12). Overall, the behaviour of $s_{\text {rel }}$ is less systematic than that of the linear correlation coefficient. The power law-behaviour found for the latter can be seen here,
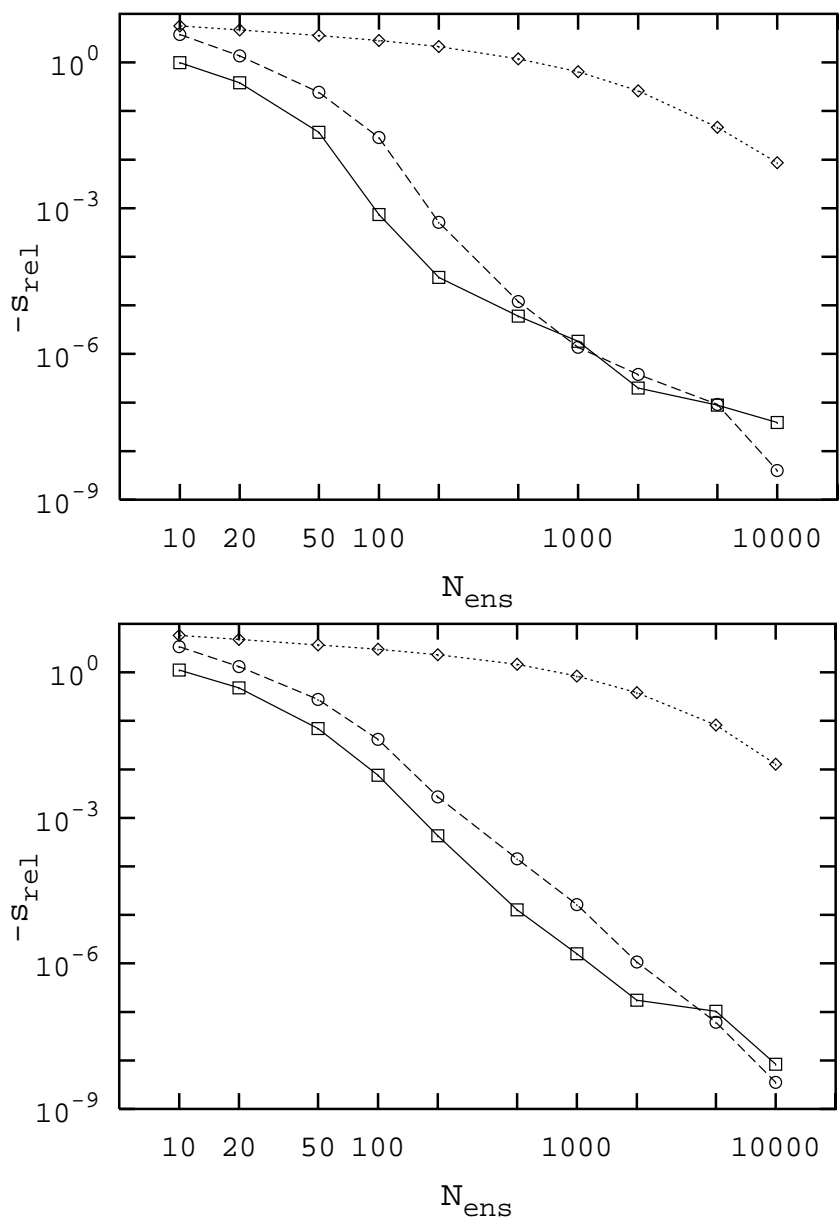

Fig. 14. Relative entropy $s_{\text {rel }}$ for the standard map $(K=0.6), \tau=1$, $d_{0}=0.001$ (upper panel) and $d_{0}=0.2$ (lower panel). Points are for $m_{\max }^{(\tau)}$ (squares), $m_{\mathrm{avg}}^{(\tau)}$ (circles) and $m_{\min }^{(\tau)}$ (diamonds).

too, but not as clearly. This fact can be attributed to the loss of information that occurs when one puts all data in one histogram and forgets about the spatial information. For the relative entropy, the convergence of $m_{\min }^{(\tau)}$ is almost as good as the one of $m_{\max }^{(\tau)}$, but this holds only for sufficently small $d_{0}$ and $\tau$.

In addition, a $\chi^{2}$-statistic was computed from the histograms obtained with different ensemble sizes. This turned out to behave in the same qualitative way as the relative entropy. Therefore, it is considered no more useful for our purpose than the measures already introduced.

For practical purposes, the asymptotic behaviour of the error growth factors with respect to ensemble size is not very helpful. What is helpful, however, is the actual ensemble size needed for the error to drop below a predefined margin, say, $\epsilon=10^{-3}$. Defining this error again as the deviation from the case $N_{\text {ens }}=20000$, we arrive at the values shown in Fig. 16 

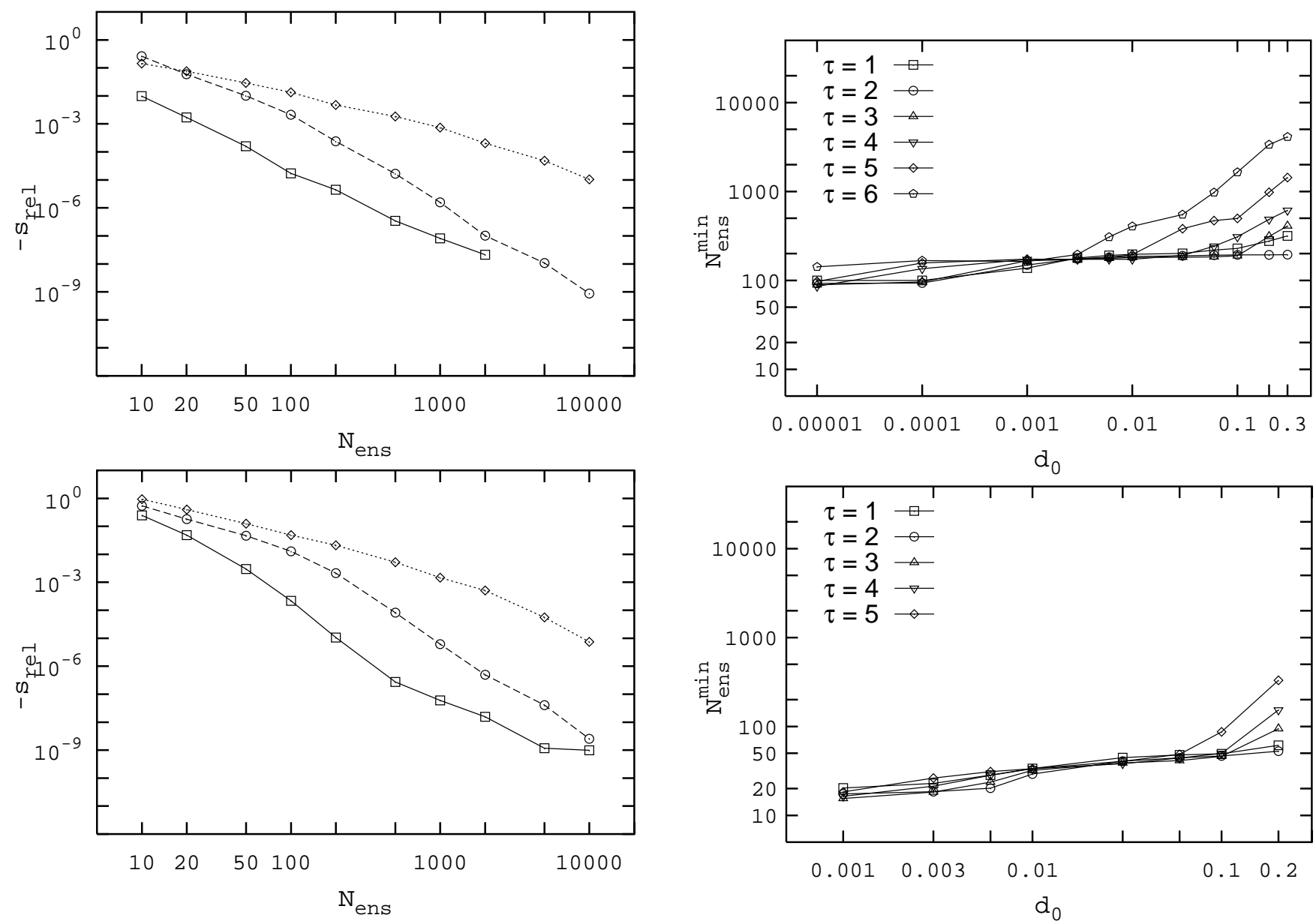

Fig. 15. As Fig. 14, but for the quasiperiodicity map $\left(c_{1}=0.42\right.$, $c_{2}=0.3, c_{3}=1.6$ ).

for $m_{\max }^{(\tau)}$. For larger initial errors $d_{0}$, one finds yet another power law,

$N_{\mathrm{ens}}^{\min } \sim d_{0}{ }^{\beta}$,

for small to intermediate prediction times $\tau$. Even smaller error margins, like $\epsilon=10^{-9}$, were also considered, but these eventually lead to a levelling off at the largest ensemble size used. In all cases considered we found a region of $d_{0}$-values indicative of the power law in Eq. (11). From the investigations done so far, a simple dependence of $\beta$ on $\tau$ or $\epsilon$ could not be established.

Apart from the systems for which results have been shown, we have also done the same studies for the Hénon map (Hénon, 1976; or see Lichtenberg and Lieberman, 1992). At the parameter values we used, $a=1.4$ and $b=0.3$, this system is strongly dissipative and exhibits a chaotic attractor. Despite these fundamental differences from the other systems considered, the same qualitative results have been found. Specifically, the scaling exponent of $1-r$ is $\gamma \approx-1$ for $m_{\text {avg }}^{(\tau)}$ and $\gamma \approx-4$ for $m_{\max }^{(\tau)}$. Regarding the minimal en-

Fig. 16. Minimal ensemble size $N_{\mathrm{ens}}^{\min }$ needed for the error in $m_{\max }^{(\tau)}$ to drop below a given margin $\epsilon$. Calculated from data as shown in Figs. 9-12, using linear interpolation in the doublelogarithmic plot. Top panel shows results for the standard map ( $K=4.2$ ) with $\epsilon<10^{-6}$, bottom panel for the quasiperiodicity map $\left(c_{1}=0.42, c_{2}=0.3, c_{3}=1.6\right)$ with $\epsilon<10^{-3}$.

semble size $N_{\text {ens }}^{\min }$, the results for the Hénon map show much less dependence on $d_{0} . N_{\text {ens }}^{\min }$ increases less than one order of magnitude for a change of $d_{0}$ from $10^{-5}$ to 0.3 and $1 \leq \tau \leq 5$.

\section{Conclusions}

We studied the growth of errors in two paradigmatic chaotic systems, the standard map and the quasiperiodicity map, that differ significantly in their distributions of local error growth factors. To this end, we followed ensembles of size $N_{\text {ens }}$ for a time $\tau$, with all ensemble members initialised with a fixed error magnitude of $d_{0}$.

We have shown that the error growth of the worst case, i.e., the largest error growth factor $M^{(\tau)}$, depends on the prediction time $\tau$ in a systematic way. For small times, there is an 
exponential increase according to the largest singular value of the linearized map. This does no longer hold if the prediction time is large. Then one observes a crossover to a regime of power-law growth, $M^{(\tau)} \sim \tau^{\alpha}$. The scaling exponent $\alpha$ of this power law has been found to depend on the size $d_{0}$ of initial errors, presumably according to a power law as well.

To find a minimal ensemble size $N_{\mathrm{ens}}^{\min }$ needed for reliable statements about predictability, we have studied various measures, namely the linear correlation coefficient $r$, the relative entropy $s_{\text {rel }}$ and the $\chi^{2}$-statistics. Here, $r$ was computed using spatially resolved data, whereas the other two were based on histograms.

For the linear correlation coefficient, a power-law has been shown to exist for large ensemble sizes, $1-r \sim N_{\text {ens }}{ }^{\gamma}$. The scaling exponent $\gamma$ is constant over a wide range of initial error sizes $d_{0}$ and prediction times $\tau$, at values of $\gamma \approx-1$ for the mean error growth and $\gamma \approx-4$ for the maximum error growth. A dependence of $\gamma$ on $d_{0}$ may only arise for $\tau$ and $d_{0}$ large enough.

Of course, this only addresses the presumably asymptotic behaviour of $\gamma$. The absolute value of the error, the error made by using a rather small ensemble, need not directly relate to the value of $\gamma$. For the cases studied herein, the maximum error growth $m_{\max }^{(\tau)}$ is typically the one converging most quickly. It can be reliably estimated with only intermediate ensemble sizes. Or, conversely, for a given error margin $\epsilon$, the minimal ensemble size $N_{\text {ens }}^{\mathrm{min}}$ is not very large.

As alternatives to ensembles, predictability can be described by local divergence rates calculated from the linearised system. Different definitions of such rates are in use, like local Lyapunov exponents $\lambda$ or maximum growth exponents $\rho$, the latter being defined on the basis of singular values $\sigma$ of the Jacobian. For very small errors $d_{0}$ and short prediction times $\tau$, one expects ensemble studies and local exponents to yield the same results. However, the choice of local exponent does matter, as different definitions can lead to different outcomes. The local Lyapunov exponents, for example, show a connection to other dynamical properties of the system such as homoclinic tangencies (Harle and Feudel, 2005). On the other hand, the maximum growth exponents are a purely local quantity, in the sense that they depend only on the systems states visited during the time span $\tau$. Since they are defined through the singular values, there is a clear correspondence between $m_{\max }^{(\tau)}$ and the largest maximum growth exponent for the same initial state. This correspondence breaks down, as expected, when $d_{0}$ or $\tau$ becomes too large.

In conclusion, we note that the determination of a minimal ensemble size for a given purpose and, consequently, for a given acceptable error is always system-dependent. In practice, the choice of ensemble size is often based on feasibility and experience. There does not seem to be any hard and fast rule about this. Still, the behaviour of the scaling exponent $\gamma$ is a general one that can be expected to be found in other sys- tems as well. Furthermore, minimal ensemble sizes needed for a given error margin can be estimated using a few ensembles at small prediction times $\tau$ and typical error values $d_{0}$. These findings can be of help in deciding on the usefulness of increasing the ensemble size.

Acknowledgements. We wish to thank J. Kurths, A. Politi and M. Zaks for helpful discussions. We also thank the referees and the editor for comments and suggestions that helped to improve the readability of this paper. Financial support from GKSS is gratefully acknowledged.

Edited by: O. Talagrand

Reviewed by: J. Anderson and another referee

\section{References}

Buizza, R.: Optimal perturbation time evolution and sensitivity of ensemble prediction to perturbation amplitude, Quart. J. Roy. Meteorol. Soc., 121, 1705-1738, 1996.

Chirikov, B. V.: A universal instability of many-dimensional oscillator systems, Phys. Rep., 52, 263-379, 1979.

Fraedrich, K., Ziehmann, C., and Sielmann, F.: Estimates of Spatial Degrees of Freedom, J. Climate, 8, 361-369, 1995.

Grebogi, C., Ott, E., and Yorke, J.: Attractors on an $N$-torus: Quasiperiodicity versus Chaos, Physica D, 15, 354-373, 1985.

Harle, M. and Feudel, U.: On the relation between predictability and homoclinic tangencies, Int. J. Bif. Chaos, 15, 2523-2534, 2005.

Hénon, M.: A two-dimensional mapping with a strange attractor, Commun. Math. Phys., 50, 69-77, 1976.

Honerkamp, J.: Stochastic Dynamical Systems, Wiley-VCH, 1994.

Kalnay, E.: Atmospheric modeling, data assimilation and predictability, Cambridge University Press, 2002.

Lichtenberg, A. J. and Lieberman, M. A.: Regular and Chaotic Dynamics, Springer, Berlin, second edn., 1992.

Mu, M., Duan, W. S., and Wang, B.: Conditional nonlinear optimal perturbation and its applications, Nonlin. Processes Geophys., 10, 493-501, 2003, mboxhttp://www.nonlin-processesgeophys.net/10/493/2003/.

Patil, D. J., Hunt, B. R., Kalnay, E., Yorke, J. A., and Ott, E.: Local Low Dimensionality of Atmospheric Dynamics, Phys. Rev. Lett., 86, 5878-5881, 2001

Smith, L. A., Ziehmann, C., and Fraedrich, K.: Uncertainty dynamics and predictability in chaotic systems, Quart. J. Roy. Meteorol. Soc., 125, 2855-2886, 1999.

Toth, Z. and Kalnay, E.: Ensemble forecasting at NMC: the generation of perturbations, Bull. Amer. Meteorol. Soc., 74, 2317 2330, 1993.

Toth, Z. and Kalnay, E.: Ensemble Forecasting at NCEP and the Breeding Method, Mon. Wea. Rev., 125, 3297-3319, 1997.

Ziehmann, C., Smith, L. A., and Kurths, J.: Localized Lyapunov exponents and the prediction of predictability, Phys. Lett. A, 271, 237-251, 2000. 